\title{
Prognostic significance of mild aortic regurgitation in predicting mortality after transcatheter aortic valve replacement
}

Brandon M. Jones, MD, ${ }^{a}$ E. Murat Tuzcu, MD, ${ }^{a}$ Amar Krishnaswamy, MD, ${ }^{a}$ Zoran Popovic, MD, Stephanie Mick, MD, ${ }^{\mathrm{b}}$ Eric E. Roselli, MD, ${ }^{\mathrm{b}}$ Sajjad Gul, MD, ${ }^{\mathrm{a}}$ Jasneet Devgun, BS, ${ }^{\mathrm{a}}$ Sohi Mistry, Wael A. Jaber, MD, ${ }^{a}$ Lars G. Svensson, MD, ${ }^{b}$ and Samir R. Kapadia, MD ${ }^{\mathrm{a}}$

\begin{abstract}
Objective: Moderate to severe aortic regurgitation after transcatheter aortic valve replacement is associated with worse outcomes. The impact of mild aortic regurgitation has been less clear, possibly because of the broad categories that have been used in clinical trials, but holds increasing importance in the study of next-generation devices in low- and intermediate-risk cohorts. A more granular scheme, which is common in clinical practice and proposed for future trials, may add prognostic value.
\end{abstract}

Methods: We evaluated all patients undergoing transfemoral transcatheter aortic valve replacement at the Cleveland Clinic from 2006 to 2012. The degree of aortic regurgitation after transcatheter aortic valve replacement was reported from the echocardiography database based on a clinical, transthoracic echocardiogram performed within 30 days of the procedure. Aortic regurgitation was finely discriminated on the basis of a multiwindow, multiparametric, integrative approach using our usual clinical scale: none, trivial to $1+, 1+, 1$ to $2+, 2+, 2$ to $3+, 3+, 3$ to $4+$, or $4+$.

Results: There were 237 patients included in the analysis. By controlling for age, gender, Society of Thoracic Surgeons score, baseline ejection fraction, and aortic regurgitation before transcatheter aortic valve replacement, there was a significant increase in mortality for each half grade of aortic regurgitation compared with the complete absence of aortic regurgitation after transcatheter aortic valve replacement. The unit hazard ratio for each $1+$ increase in aortic regurgitation after transcatheter aortic valve replacement was 2.26 (95\% confidence interval, 1.48-3.43; $P<.001)$ considering aortic regurgitation as a continuous variable. Other clinical variables did not significantly affect mortality.

Conclusions: Even mild aortic regurgitation after transcatheter aortic valve replacement is associated with worse long-term mortality. There may be prognostic value in reporting milder categories of aortic regurgitation with more granular gradations. (J Thorac Cardiovasc Surg 2016;152:783-90)

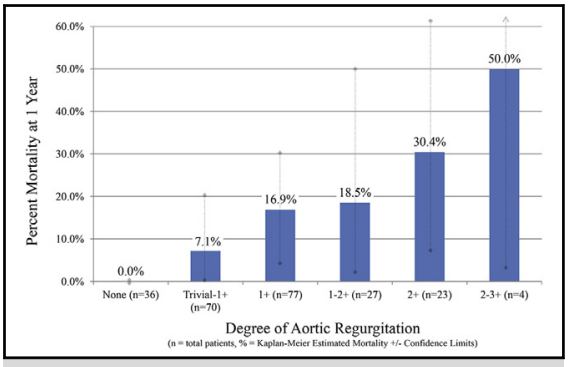

Mortality at 1 year with varying degrees of AR after TAVR.

\section{Central Message}

A more granular characterization of mild $\mathrm{AR}$ after TAVR is effective in predicting longterm mortality and will hold increasing importance as the technology transitions to low-risk populations.

\section{Perspective}

A more granular, clinical grading scheme for grading AR after TAVR can effectively predict mortality, and even trivial AR was independently associated with risk-adjusted, longterm mortality. Therefore, there may be prognostic value in reporting milder categories of AR with more granular gradations, compared with the traditional, broad categories used in the current TAVR literature.

See Editorial page 659.
Transcatheter aortic valve replacement (TAVR) is an effective treatment for high-risk and inoperable patients with severe aortic stenosis (AS). ${ }^{1-4}$ As the technology expands, especially to intermediate-risk populations, there is great

\footnotetext{
From the Departments of ${ }^{\mathrm{a} C a r d i o v a s c u l a r}$ Medicine and ${ }^{\mathrm{b}}$ Cardiothoracic Surgery, Cleveland Clinic Foundation, Cleveland, Ohio.

Received for publication Feb 3, 2016; revisions received April 26, 2016; accepted for publication May 9, 2016; available ahead of print June 16, 2016

Address for reprints: Samir R. Kapadia, MD, Department of Cardiovascular Medicine, Cleveland Clinic, 9500 Euclid Ave, Desk J2-3, Cleveland, OH 44195 (E-mail: kapadis@ccf.org).

$0022-5223 / \$ 36.00$

Copyright (c) 2016 by The American Association for Thoracic Surgery http://dx.doi.org/10.1016/j.jtcvs.2016.05.023
}

focus on reducing procedural complications, including paravalvular leak (PVL). ${ }^{5,6}$ Many studies have shown an increase in the incidence of PVL among patients undergoing TAVR compared with surgical aortic valve replacement and a significant increase in mortality among those patients with moderate or severe aortic regurgitation

Scanning this QR code will take you a video for the article.

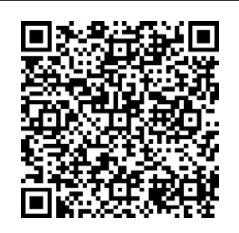




$\begin{array}{ll}\text { Abbreviations and Acronyms } \\ \text { AR } & =\text { aortic regurgitation } \\ \text { AS } & =\text { aortic stenosis } \\ \text { ASE } & =\text { American Society of } \\ & \text { Echocardiography } \\ \text { CI } & =\text { confidence interval } \\ \text { HR } & =\text { hazard ratio } \\ \text { PARTNER }= & \text { Placement of Aortic Transcatheter } \\ & \text { Valves } \\ \text { PVL } & =\text { paravalvular leak } \\ \text { STS } & =\text { Society of Thoracic Surgeons } \\ \text { TAVR } & =\text { transcatheter aortic valve replacement } \\ \text { VARC } & =\text { Valve Academic Research Consortium }\end{array}$

(AR) after TAVR. ${ }^{7-13}$ However, the impact of milder forms of AR after TAVR has been less clear. ${ }^{12,14,15}$ One limitation in the understanding of the relationship between AR and mortality after TAVR has been inconsistencies in how AR is categorized between different studies and core laboratories. There is disagreement on the most appropriate imaging modality to accurately judge AR (echocardiography, cardiac magnetic resonance, invasive hemodynamics, angiography) and further disagreement within each modality regarding the guidelines for grading AR. ${ }^{16-19}$

Traditionally, echocardiography core laboratory adjudication of AR after TAVR has followed a broad, 3-class scale (mild, moderate, or severe) based on the principles endorsed by the American Society of Echocardiography (ASE) and Valve Academic Research Consortium (VARC-2) guidelines. ${ }^{17,19}$ Because of these broad categorizations, however, it is not well understood how milder forms of AR affect long-term outcomes after TAVR, and "trivial" AR traditionally has been lumped together with "none" in contemporary TAVR trials. Severity is traditionally judged on the basis of a multiparametric approach that combines information from multiple echocardiographic views and contains some inherent subjectivity making broad, rigid characterizations difficult. Recently, a 5-class scheme (adding mild-moderate and moderate-severe) has been proposed that would allow for "between-grade" options, with the hypothesis that a more granular strategy would allow for better prognostic discrimination than when only provided with 3 options, but proponents of this strategy admit that there is currently a lack of outcomes data to support a more granular approach. ${ }^{20}$ Finally, a 7-class scale for grading AR in clinical trials has been proposed (adding "none" and "trivial"), but also has not been proven to add prognostic value. ${ }^{16}$ Outside of major clinical trials and core laboratories, many academic institutions use a standard, clinical grading scale for AR based on a 0 to $4+$ scheme. ${ }^{21}$ Although not expressly endorsed by major society guidelines or widely described in the literature, this grading system represents a real-world approach to grading AR after TAVR. Furthermore, by allowing for "between-grade" options, this permits AR to be finely categorized as none, trivial to $1+$, $1+, 1$ to $2+, 2+, 2$ to $3+, 3+, 3$ to $4+$, or $4+$.

The aim of our study was to evaluate the ability of a more granular, clinical grading scheme for characterizing AR to predict outcomes after TAVR. Our secondary goal was to understand the long-term impact of the entire spectrum of AR, but especially more mild degrees, on mortality after TAVR.

\section{MATERIALS AND METHODS}

We retrospectively evaluated all patients undergoing transfemoral TAVR with the first-generation Edwards SAPIEN or SAPIEN-XT balloon-expandable bioprosthetic valve (Edwards Lifesciences, Irvine, Calif) at the Cleveland Clinic from May 2006 to December 2012. Patients undergoing TAVR via thoracic or other nontraditional access routes were excluded to reduce potentially confounding variables. This study was approved by the institutional review board at the Cleveland Clinic. All procedures were completed by 2 experienced operators with only a single intraprocedural death. Patients were excluded if they died during the procedure, did not have a valve deployed during the procedure, required conversion to a transapical or open surgical valve replacement, or were lost to follow-up after the index hospitalization. Baseline characteristics, including age, gender, Society of Thoracic Surgeons (STS) score, ejection fraction, and AR before TAVR, were obtained on the basis of review of the electronic medical record and the echocardiogram database. Mortality was confirmed on the basis of review of the electronic medical record or yearly phone calls from a research coordinator for patients who were followed at another institution. The primary end point (survival) was censored to the last known date of confirmed survival for each patient.

The degree of AR after TAVR was obtained from the echocardiogram database based on a clinical, transthoracic echocardiogram completed within 30 days of the procedure. AR was graded on a clinical scale ranging from "none" to "4+", which further allowed for between-grade scoring. Grading was multiparametric and integrative, and emphasized the quantitative and qualitative parameters endorsed by the ASE and VARC-2 guidelines. The following data were routinely obtained to determine the severity of AR: Left ventricular size was classified as normal or dilated according to the ASE guidelines on native valvular regurgitation; the regurgitant jet was evaluated for flow convergence, vena contracta, and jet area at a Nyquist limit of 50 to $60 \mathrm{~cm} / \mathrm{s}$ in parasternal long-axis and apical views; the circumferential extent of the regurgitant jet was evaluated in the parasternal short-axis view; jet density and pressure halftime were evaluated by continuous-wave Doppler in the best aligned views; and diastolic flow reversal was evaluated by pulse-wave Doppler in the descending thoracic aorta. On the basis of a combination of these data points, each echocardiogram was assigned a grade of AR by the interpreting echocardiography staff. The grade assigned at the time of clinical interpretation was recorded from the echocardiography database, and no studies were corrected or reread by study investigators. The grade of AR reported represented paravalvular $\mathrm{AR}$, and there were no cases in which there was significant valvular regurgitation that contributed to the total grade. By convention, "none" was considered the complete absence of discernible AR; trivial to $1+, 1+$, and 1 to $2+$ were considered gradations of mild AR; $2+, 2$ to $3+$, and $3+$ were considered gradations of moderate AR; and 3 to $4+$ and $4+$ were considered severe. Echocardiograms were interpreted by approximately 25 individual level 3 certified echocardiographers who are also involved in the interpretation of PVL for the echocardiography CoreLab at the Cleveland Clinic. Although not specific to this study, 
variability analysis for the grading of PVL after TAVR is conducted as part of an ongoing, semiannual process in our CoreLab. For the 7-class scale used by the CoreLab, the kappa was 0.89 for interobserver variability and 0.91 for intraobserver variability, with no cases resulting in more than 1 grade of reclassification.

Simple descriptive statistics are used to summarize the data. Continuous variables are presented as mean \pm standard deviation and as 25th, 50th (median), and 75th percentiles for skewed data. Categoric variables are described using frequencies and percentages. Survival is estimated nonparametrically by the Kaplan-Meier estimator. Risk-adjusted impact of post-TAVR AR is assessed using Cox proportional hazard regression, including age, gender, STS score, baseline ejection fraction, and preTAVR AR in the Cox multivariable regression model. $P$ values are reported as hazard ratios (HRs) with $95 \%$ confidence intervals (CIs). All analyses were performed using JMP Pro statistical software from SAS (version 10.0.0; SAS Institute Inc, Cary, NC).

\section{RESULTS}

There were 255 patients who underwent attempted transfemoral TAVR during the study period. Eighteen patients were excluded from the analysis (1 patient died during the procedure, 3 procedures were converted to transapical TAVR, 8 procedures were aborted, 2 patients required emergency surgical valve replacement, and 4 patients were lost to follow-up after the index hospitalization). Baseline characteristics for the remaining 237 patients are listed in Table 1. On the basis of a clinical transthoracic echocardiogram within 30 days of the procedure, paravalvular AR was graded as none $(\mathrm{n}=36,15.2 \%)$, trivial to $1+(\mathrm{n}=70$, $29.5 \%), 1+(\mathrm{n}=77,32.5 \%), 1$ to $2+(\mathrm{n}=27,11.4 \%)$, $2+(\mathrm{n}=23,9.7 \%)$, or 2 to $3+(\mathrm{n}=4,1.7 \%)$ as shown in Figure 1. There were no patients with $3+$ or greater AR within 30 days of TAVR. There were no patients with significant valvular AR immediately after TAVR. PreTAVR AR also was measured by clinical transthoracic echocardiogram at an average of $29 \pm 43$ days before the procedure. Pre-TAVR AR was graded as none $(\mathrm{n}=18$, $7.6 \%)$, trivial to $1+(\mathrm{n}=67,28.3 \%), 1+(\mathrm{n}=81$, $34.2 \%), 1$ to $2+(\mathrm{n}=26,11.0 \%), 2+(\mathrm{n}=36,15.2 \%)$, 2 to $3+(\mathrm{n}=2,0.8 \%), 3+(\mathrm{n}=6,2.5 \%)$, or 3 to $4+$ $(\mathrm{n}=1,0.4 \%)$ as shown in Figure 1 .

For the overall cohort, there were 61 deaths over a mean follow-up period of $909( \pm 534)$ days. The median followup time was 824 days, and $10 \%$ of the survivors were followed for more than 4 years. In patients with no AR after TAVR, there was $0 \%$ mortality at 1 year, and with each subsequent grade of AR there was an increase in 1-year mortality. The 1-year, Kaplan-Meier estimated mortality of patients based on the degree of AR after TAVR is depicted in Figure 2. Kaplan-Meier survival over the entire period of follow-up based on the degree of AR after TAVR is shown in Figure 3. By controlling for age, gender, STS score, baseline left ventricular ejection fraction, and AR before TAVR, there was a significant increase in mortality associated with each half grade of AR compared with "none" (HR for trivial-1+ AR, 4.4 [95\% CI, 1.2-27.7], $P=.02$; HR for
TABLE 1. Baseline characteristics

\begin{tabular}{lc}
\hline Total patients & $\mathrm{n}=237$ \\
Age & $80.6 \pm 10.0 \mathrm{y}$ \\
Male & $58.2 \%$ \\
STS score & $9.7 \% \pm 5.1 \%(25$ th $6.1 \%$, \\
& median $9.2 \%, 75$ th $11.7 \%)$ \\
Baseline LVEF & $49.5 \% \pm 14.3 \%$ \\
Diabetes & $44.3 \%$ \\
Hyperlipidemia & $76.7 \%$ \\
Hypertension & $84.8 \%$ \\
CAD & $83.1 \%$ \\
Creatinine & $1.16 \pm 0.45 \mathrm{~g} / \mathrm{dL}(25$ th 0.88, \\
& median $1.07,75$ th 1.34$)$ \\
Prior CABG & $48.1 \%$ \\
NYHA class III/IV & $93.7 \%$ \\
\hline
\end{tabular}

$S T S$, Society of Thoracic Surgeons; $L V E F$, left ventricular ejection fraction; $C A D$, coronary artery disease; $C A B G$, coronary artery bypass grafting; $N Y H A$, New York Heart Association.

$1+$ AR, 4.6 [95\% CI, 1.3-29.3], $P=.01$; HR for 1 to $2+$ AR, 6.4 [95\% CI, 1.7-41.7], $P=.005$; HR for $2+\mathrm{AR}$, 9.7 [95\% CI, 2.4-64.1], $P=.0008$ ) (Table 2). Of the 4 patients with 2 to $3+\mathrm{AR}$, there were 2 deaths at 1 year. There was no significant difference in mortality based on age, gender, STS score, or baseline ejection fraction in the multivariate model or when tested as individual variables. As a secondary, confirmatory analysis, multivariate Cox regression analysis was completed using "AR post-TAVR" as a continuous variable (Table 3) and demonstrated the unit HR for each $1+$ increase in AR after TAVR to be 2.26 (95\% CI, 1.48-3.43; $P<.001$ ). On subsequent univariate analysis, pre-TAVR left ventricular internal diastolic diameter was not associated with post-TAVR mortality $(P=.98)$, nor was pre-TAVR baseline creatinine value $(P=.52)$.

To show consistency between the more granular scheme and the standard 3-class scheme, we collapsed our data down into the traditional, broad categories of none $(\mathrm{n}=36$ ), mild (trivial- $1+, 1+$, and 1 to $2+; \mathrm{n}=174)$, moderate $(2+, 2$ to $3+$, and $3+; n=27)$, and severe ( 3 to $4+$ and $4+; \mathrm{n}=0$ ). Again, adjusting for age, gender, STS score, baseline ejection fraction, and AR before TAVR, there was a significant increase in mortality for both mild AR (HR, 4.8; 95\% CI, 1.5-29.7; $P=.005$ ) and moderate AR (HR, 11.5; 95\% CI, 3.1-74.9; $P<.0001$ ) compared with none, and with moderate compared with mild AR (HR, 2.4; 95\% CI, 1.2-4.5; $P=.02$ ) (Figure $4, A$ and $B$ ).

In all cases, AR was evaluated at the time of valve implantation by transesophageal echocardiography, root angiography, hemodynamic assessment, or a combination of these modalities. The most common reasons for acute AR were PVL from the valve being deployed too high in the annulus or from asymmetric calcification, or rarely valvular AR from restricted leaflet motion ( 2 cases, both treated with placement of a second valve). When significant AR was discovered, a number of strategies were used to attempt to 


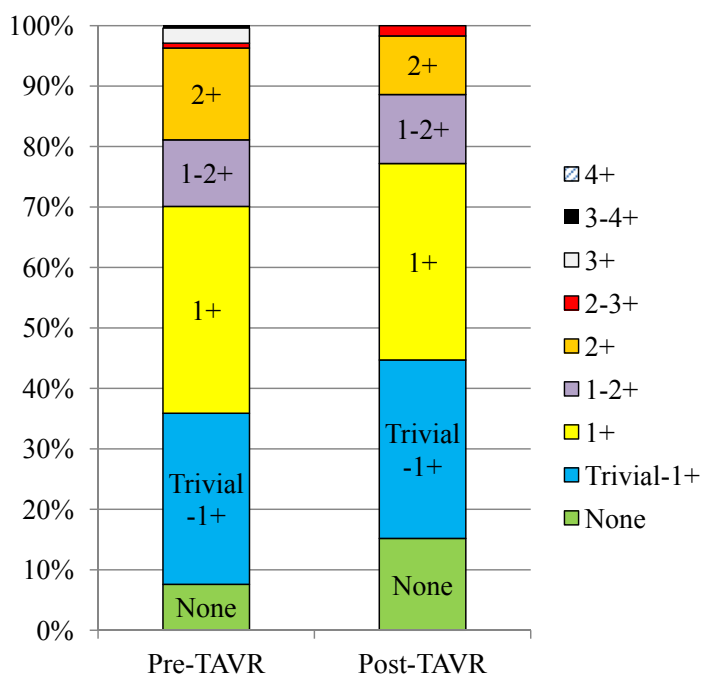

\begin{tabular}{c|cc} 
& Pre-TAVR & Post-TAVR \\
\hline None & $7.6 \%$ & $15.2 \%$ \\
\hline Trivial-1+ & $28.3 \%$ & $29.5 \%$ \\
\hline $1+$ & $34.2 \%$ & $32.5 \%$ \\
\hline $1-2+$ & $11.0 \%$ & $11.4 \%$ \\
\hline $2+$ & $15.2 \%$ & $9.7 \%$ \\
\hline $2-3+$ & $0.8 \%$ & $1.7 \%$ \\
\hline $3+$ & $2.5 \%$ & None \\
\hline $3-4+$ & $0.4 \%$ & None \\
\hline $4+$ & none & None \\
\hline
\end{tabular}

FIGURE 1. Incidence and severity of AR before and after TAVR based on a clinical, echocardiographic grading scheme. TAVR, Transcatheter aortic valve replacement.

reduce the severity. In 14 patients $(5.9 \%)$, balloon postdilatation was used to improve the degree of regurgitation. In 9 patients $(3.8 \%)$, an additional valve was placed inside the original valve, with 1 such patient requiring a total of 3 valves. Two patients $(0.8 \%)$ required an additional valve after balloon dilatation, but this did not sufficiently improve the degree of AR. One patient $(0.4 \%)$ had residual paravalvular regurgitation despite a second valve being placed, so an Amplatzer Vascular Plug II (St Jude Medical, St Paul, Minn) was placed, and the AR was improved. Video 1 shows 1 case in which paravalvular regurgitation was successfully treated with balloon postdilation.

\section{DISCUSSION}

Our study shows that characterizing AR after TAVR based on a more granular scale provides important prognostic information with regard to both 1-year and longterm mortality. Furthermore, even trivial to $1+$ AR after TAVR was independently associated with risk-adjusted mortality in a cohort of patients undergoing transfemoral, balloon-expandable TAVR. Remarkably, among the 41 patients who had no discernible AR after TAVR, there were no deaths at 1 year, despite $13.9 \%$ mortality at 1 year for the overall cohort. Although the Kaplan-Meier survival curves for trivial to $1+, 1+$, and 1 to $2+\mathrm{AR}$ appear to overlap

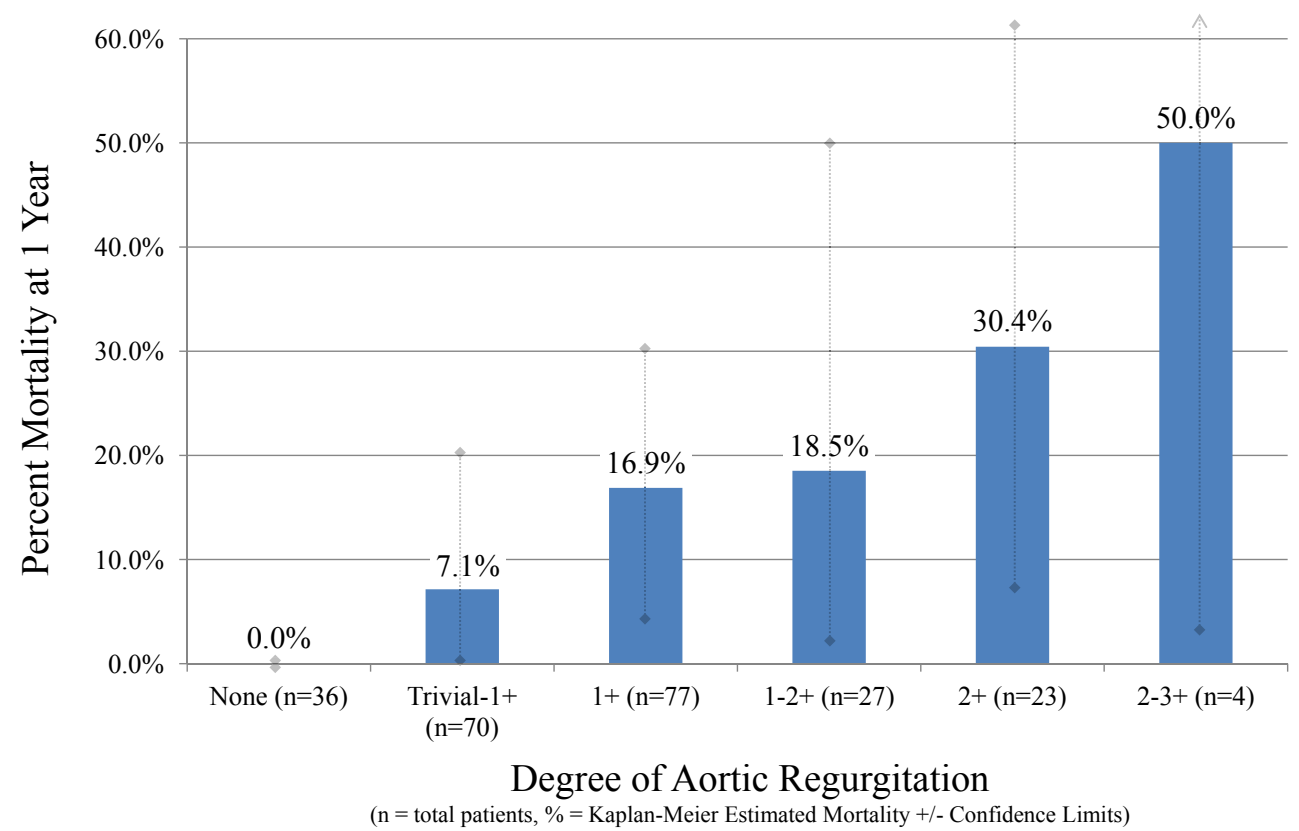

FIGURE 2. Kaplan-Meier estimated mortality at 1 year with varying degrees of AR after TAVR as judged by a clinical echocardiographic grading scheme. 


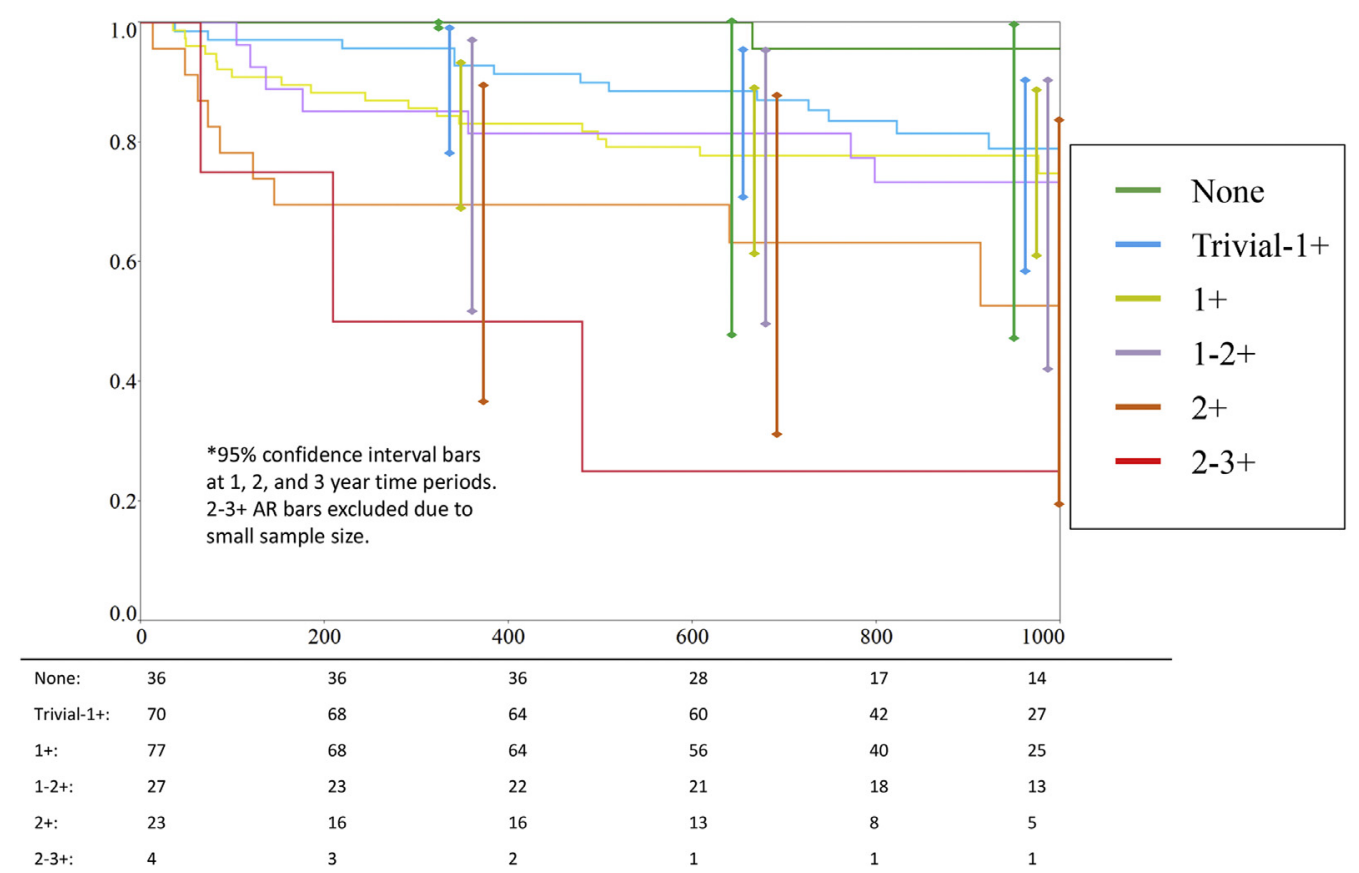

FIGURE 3. Long-term Kaplan-Meier estimated survival with varying degrees of AR after TAVR as judged by a clinical echocardiographic grading scheme with number of patients at risk at each time interval. $A R$, Aortic regurgitation.

considerably over multiple years of follow-up, there was an approximately 2 -fold increase in mortality at 1 year for patients with $1+(16.9 \%)$ and 1 to $2+(18.5 \%)$ AR, compared with those with trivial to $1+$ AR $(7.1 \%)$. The apparent stepwise increase in mortality for each grade of AR at 1 year suggests that there may be prognostic value in more fine gradations compared with the more broad 3-scale system used in the clinical trials for the Edwards SAPIEN Valve and Medtronic CoreValve (Medtronic Inc, Minneapolis, Minn).

Our findings are consistent with recent reports from the Placement of Aortic Transcatheter Valves (PARTNER) trial and registries that demonstrate an increase in 1-year, allcause mortality and rehospitalization among patients with mild versus none/trace AR. ${ }^{15}$ However, the mechanisms responsible for these findings remain unknown. It has been demonstrated that patients with moderate to severe AR after TAVR have less improvement in New York Heart

TABLE 2. Adjusted hazard ratios for mortality based on KaplanMeier survival for each clinical echocardiographic grade of aortic regurgitation compared with "none," adjusting for age, gender, Society of Thoracic Surgeons score, aortic regurgitation pretranscatheter aortic valve replacement, and left ventricular ejection fraction pretranscatheter aortic valve replacement

\begin{tabular}{ll}
\hline None vs trivial to $1+\mathrm{AR}$ & $\mathrm{HR}, 4.4(95 \% \mathrm{CI}, 1.2-27.7 ; P=.02)$ \\
None vs $1+\mathrm{AR}$ & $\mathrm{HR}, 4.6(95 \% \mathrm{CI}, 1.3-29.3 ; P=.01)$ \\
None vs 1 to $2+\mathrm{AR}$ & $\mathrm{HR}, 6.4(95 \% \mathrm{CI}, 1.7-41.7 ; P=.005)$ \\
None vs $2+\mathrm{AR}$ & $\mathrm{HR}, 9.7(95 \% \mathrm{CI}, 2.4-64.1 ; P=.0008)$ \\
None vs 2 to $3+\mathrm{AR}$ & 4 patients, 2 deaths at $1 \mathrm{y}$ \\
\hline
\end{tabular}

$A R$, Aortic regurgitation; $H R$, hazard ratio; $C I$, confidence interval.
Association class at 6 months compared with patients with none or mild AR, and that patients with moderate to severe AR have less improvement in left ventricle volumes and left ventricle mass index, suggesting plausible links between AR and mortality. ${ }^{15}$ Accordingly, it should be pointed out that all forms of moderate AR are not equivalent, and that as moderate AR approaches severe, there seems to be an increasing impact on outcomes, which may be driven by these mechanisms.

Unfortunately, our study continues to fall short of identifying the mechanisms that are responsible for increased mortality among patients with mild PVL after TAVR, and it is not plausible that trivial AR alone would be directly responsible for increased mortality. Perhaps it is a marker for other factors that are difficult to quantify, such as a

TABLE 3. Multivariate Cox regression analysis for mortality after transcatheter aortic valve replacement with aortic regurgitation after transcatheter aortic valve replacement as the dependent variable and covariates

\begin{tabular}{lcc}
\hline \multicolumn{1}{c}{ Variable } & $\boldsymbol{P}$ value & Unit HR $(\mathbf{9 5} \% \mathbf{C I})$ \\
\hline AR post-TAVR & .0002 & $2.26(1.48-3.43)$ \\
Age & .09 & $1.03(0.99-1.06)$ \\
Gender (male) & .37 & $1.28(0.74-2.28)$ \\
STS score & .40 & $0.97(0.91-1.03)$ \\
LVEF pre-TAVR & .88 & $1.00(0.98-1.02)$ \\
AR pre-TAVR & .47 & $1.15(0.78-1.67)$ \\
\hline
\end{tabular}

Considering AR after TAVR as a continuous variable, the unit $\mathrm{HR}$ for each 1+ grade increase in AR is shown. $H R$, Hazard ratio; $C I$, confidence interval; $A R$, aortic regurgitation; TAVR, transcatheter aortic valve replacement; STS, Society of Thoracic Surgeons; $L V E F$, left ventricular ejection fraction. 

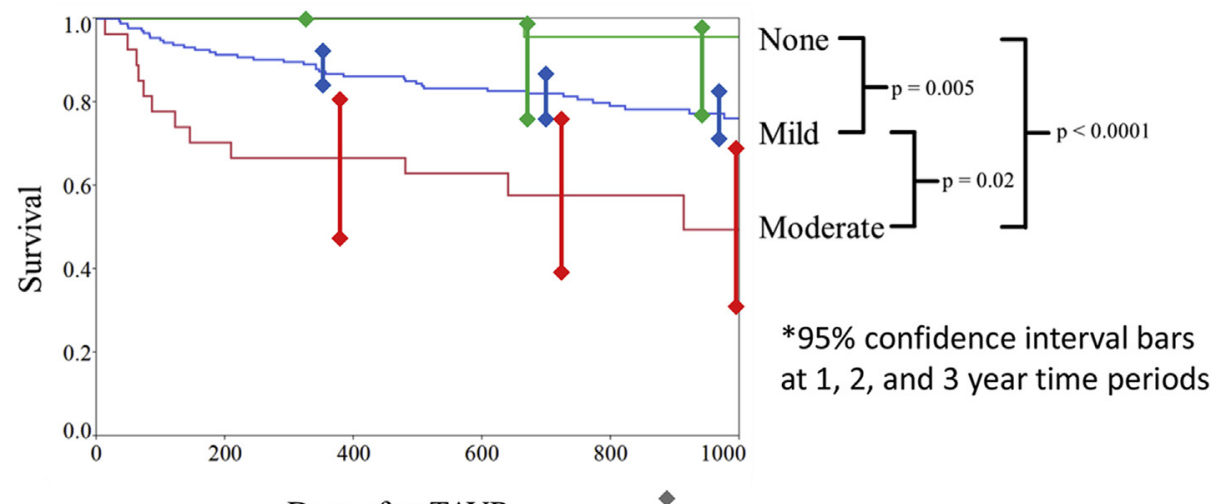

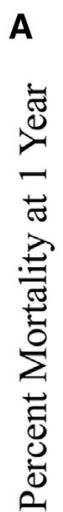

B

Days after TAVR

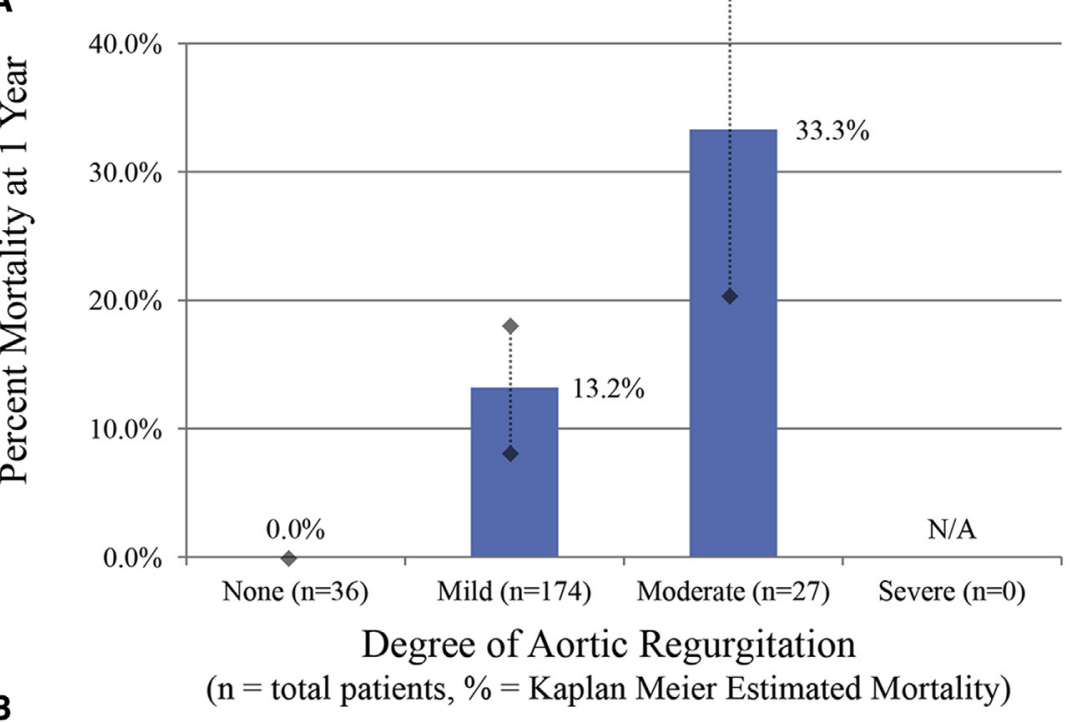

FIGURE 4. Long-term Kaplan-Meier survival (A) and 1-year Kaplan-Meier estimated mortality (B) based on a collapsed 3-scale grading scheme for AR after TAVR. Mild includes trivial to $1+, 1+$, or 1 to $2+$. Moderate includes $2+, 2$ to $3+$, or $3+$. Severe includes 3 to $4+$ and $4+. P$ values based on adjusted HRs comparing groups in the multivariable Cox proportional hazard model. N/A, Not available; TAVR, transcatheter aortic valve replacement.

highly calcified or asymmetric annulus, or an unwillingness to aggressively postdilate the valve because of other procedural factors. Another explanation is that the signal for increased mortality with even trivial AR in our study could be a reflection of underestimation of AR by echocardiography, as recently discussed in the review by Pibarot and colleagues. ${ }^{20}$ This is important because recent data from the PARTNER 2 and S3i trials have demonstrated extremely low rates of moderate or severe AR after TAVR and failed to show a difference in 1-year mortality between patients with no or trivial AR compared with mild AR. ${ }^{5}$ However, there was a $22.5 \%$ incidence of mild PVL in the PARTNER 2 trial, and despite no differences in outcomes between these patients and those with no or trivial AR after TAVR, perhaps a difference was somewhat obscured by the separation of patients with trivial AR from the "mild" group. Certainly, a more comprehensive understanding of the spectrum of trivial to mild AR now takes on increasing importance, with the significant reductions in moderate to severe PVL among the current generation of TAVR devices and as studies extend to low-risk populations.

Unique to our study is the fact that the echocardiographic data were derived from real clinical practice. Although the clinical grading scale we report is the system that many, if not most, academic centers in the United States use to classify AR in clinical practice, it clearly lacks the prior validation and major society endorsement that are present for the traditional 3-class grading scheme. That said, this was an integrative assessment that did not emphasize or rely exclusively on any single parameter, which is consistent with the recommendations of the VARC-2 and ASE guidelines, and is in harmony with a recent report by Hahn and colleagues, ${ }^{22}$ recommending an integrative approach for the assessment of valvular regurgitation post-TAVR (Figure 5)..$^{17,19}$

Despite the single-center nature of our report, the number of physicians interpreting the echocardiograms was in excess of 25 , all level 3 certified echocardiographers. Even with this potential for intraobserver and interobserver 


\begin{tabular}{|c|c|c|c|c|c|c|c|c|}
\hline & \multirow[t]{2}{*}{ None } & Trivial & $1+$ & $1-2+$ & $2+$ & $2-3+$ & $3+$ & $3-4+$ \\
\hline & & \multicolumn{3}{|c|}{ Gradations of Mild AR } & \multicolumn{3}{|c|}{ Gradations of Moderate AR } & Gradations of severe AR \\
\hline $\begin{array}{l}\text { Circumferential extent of } \\
\text { the Regurgitant Jet } \\
\text { (Short Axis) }\end{array}$ & $\mathrm{n} / \mathrm{a}$ & \multicolumn{2}{|c|}{$<10 \%$} & & \multicolumn{3}{|c|}{$10-30 \%$} & $>30 \%$ \\
\hline Pressure Halftime & $\mathrm{n} / \mathrm{a}$ & \multicolumn{3}{|c|}{$>500 \mathrm{~ms}$} & \multicolumn{3}{|c|}{$200-500 \mathrm{~ms}$} & $<200 \mathrm{~ms}$ \\
\hline Jet Density & $\mathrm{n} / \mathrm{a}$ & \multicolumn{2}{|c|}{ Faint } & Inte & & \multicolumn{3}{|c|}{ Dense } \\
\hline Diastolic Flow Reversal & \multicolumn{2}{|c|}{ Absent } & \multicolumn{3}{|c|}{ Brief / Early diastolic } & \multicolumn{2}{|c|}{ Intermediate } & Holodiastolic \\
\hline Regurgitant Fraction & $\mathrm{n} / \mathrm{a}$ & \multicolumn{3}{|c|}{$<30 \%$} & \multicolumn{3}{|c|}{$30-49 \%$} & $\geq 50 \%$ \\
\hline LV Size & \multicolumn{4}{|c|}{ Usually Normal } & \multicolumn{3}{|c|}{ Mild Dilation } & Dilated \\
\hline
\end{tabular}

FIGURE 5. Representative, quantitative variables used in the grading of aortic regurgitation after transcatheter aortic valve replacement based on a more granular, clinical grading scale as adapted from American Society of Echocardiography and Valve Academic Research Consortium guidelines. Note that these are not rigid rules but guidelines, and between-grade options are useful for grading cases with discordant data. $A R$, Aortic regurgitation; $n / a$, not available; $L V$, left ventricle.

variability, a strong signal for mortality was detected with each clinical grade of AR. Furthermore, the risk of substantial variability among the echocardiography staff who clinically interpreted these studies is likely low given that these individuals routinely interpret PVL for our CoreLab, which has documented excellent intraobserver and interobserver variability for grading paravalvular AR after TAVR using a 7-class scale. However, it remains to be proven if sitereported AR correlates with core laboratory-reported severity of AR among patients in the PARTNER trial and how strongly each method of grading is associated with

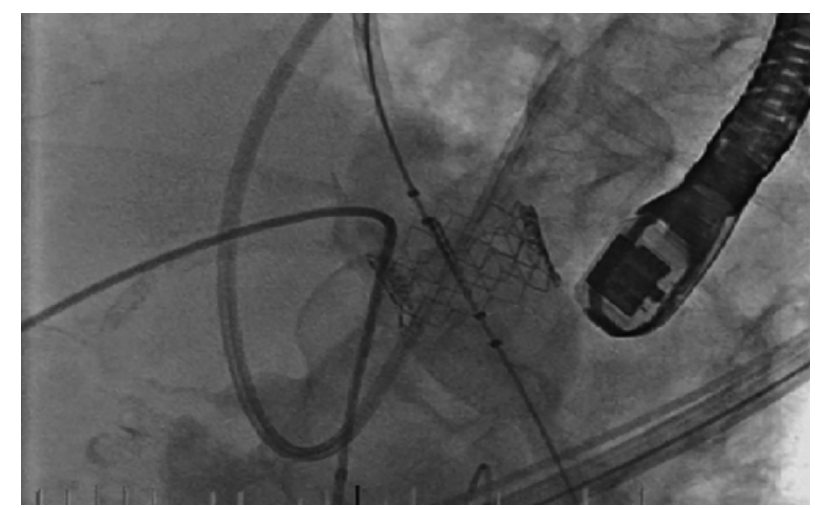

VIDEO 1. Management of acute paravalvular AR after TAVR. This video shows implantation of a \#23 Edwards SAPIEN (Edwards Lifesciences, Irvine, Calif) aortic valve in an 88 -year-old woman with severe AS. After valve deployment, there is severe AR as seen by transesophageal echocardiogram and by near equalization of the aortic diastolic and left ventricle end-diastolic pressures. Balloon postdilation is completed, and there is only trivial residual paravalvular regurgitation as seen by transesophageal echocardiogram, hemodynamic evaluation, and root aortography. Video available at http://www.jtcvsonline.org/article/S0022-5223(16)30461-5/ addons. clinical outcomes. Of note, it is not our intention to present our grading scale as an equivalent or a replacement for the 3 -class scale that is traditionally used in clinical trials. Simply, we want to provide proof that when provided with a system that allows for more fine gradations for quantifying AR, there is additional discriminant value. We believe that this is an important contribution to the literature, especially as conversations are ongoing about transitioning to a 7-grade scale for future TAVR trials and registries.

\section{Study Limitations}

The major limitations of our study include the fact that it is a single-center experience with site-reported AR. Furthermore, the clinical grading scale used in this study, although widely used in clinical practice, has never been specifically validated for interobserver and intraobserver variability. Another limitation was that our study included small numbers of patients with greater than $1+$ AR and no patients with severe AR after TAVR, which is likely responsible for the large CIs reported. In addition, we evaluated patients undergoing early-generation SAPIEN, balloon-expandable TAVR, which has been replaced in clinical practice by newer-generation devices. To test the concept of a more granular scale for grading AR though, we needed to select a population with a significant incidence of PVL, in whom we had long-term followup data and in whom there were enough primary end points to show statistical significance, all of which favored focusing on an early-generation cohort. How this type of a clinical scheme for grading AR would perform in predicting outcomes in the CoreValve or newer-generation devices such as the SAPIEN 3 remains to be seen.

\section{CONCLUSIONS}

A more granular, clinical, real-world grading scheme for grading AR after TAVR can effectively predict mortality. In 
our study, even trivial AR after TAVR was independently associated with risk-adjusted, long-term mortality in this single, large-volume center. Understanding the impact of mild AR takes on increasing significance with the extremely low rates of moderate or severe PVL that have been reported among newer-generation TAVR devices. Therefore, there may be prognostic value in separating AR into more fine gradations compared with the traditional, broad categories used in the current TAVR literature. Further investigation is needed to understand the mechanisms responsible for these associations.

\section{Conflict of Interest Statement}

There was no funding source used for this study. The authors report no conflicts of interest directly related to the work under consideration for publication. E.M.T. receives travel reimbursement from Edwards Lifescience and DirectFlow Medical for the PARTNER and Salus trials. E.E.R. receives consulting and/or speaking fees from Edwards Lifescience, Medtronic, St Jude, and LivaNova. W.A.J. receives compensation from Edwards Lifescience for CoreLab work in the PARTNER trial. All other authors have nothing to disclose with regard to commercial support.

\section{References}

1. Leon MB, Smith CR, Mack M, Miller DC, Moses JW, Svensson LG, et al; Investigators PT. Transcatheter aortic-valve implantation for aortic stenosis in patients who cannot undergo surgery. $N$ Engl J Med. 2010;363:1597-607.

2. Smith CR, Leon MB, Mack MJ, Miller DC, Moses JW, Svensson LG, et al; Investigators PT. Transcatheter versus surgical aortic-valve replacement in highrisk patients. $N$ Engl J Med. 2011;364:2187-98.

3. Adams DH, Popma JJ, Reardon MJ, Yakubov SJ, Coselli JS, Deeb GM, et al; Investigators USCC. Transcatheter aortic-valve replacement with a self-expanding prosthesis. N Engl J Med. 2014;370:1790-8.

4. Popma JJ, Adams DH, Reardon MJ, Yakubov SJ, Kleiman NS, Heimansohn D, et al. Transcatheter aortic valve replacement using a self-expanding bioprosthesis in patients with severe aortic stenosis at extreme risk for surgery. J Am Coll Cardiol. 2014;63:1972-81.

5. Leon MB, Smith CR, Mack MJ, Makkar RR, Svensson LG, Kodali SK, et al. Transcatheter or surgical aortic-valve replacement in intermediate-risk patients. N Engl J Med. 2016;374:1609-20.

6. Iturra SA, Suri RM, Greason KL, Stulak JM, Burkhart HM, Dearani JA, et al. Outcomes of surgical aortic valve replacement in moderate risk patients: implications for determination of equipoise in the transcatheter era. J Thorac Cardiovasc Surg. 2014;147:127-32.

7. Abdel-Wahab M, Zahn R, Horack M, Gerckens U, Schuler G, Sievert H, et al Aortic regurgitation after transcatheter aortic valve implantation: incidence and early outcome. Results from the German Transcatheter Aortic Valve Interventions Registry. Heart. 2011;97:899-906.

8. Moat NE, Ludman P, de Belder MA, Bridgewater B, Cunningham AD, Young CP, et al. Long-term outcomes after transcatheter aortic valve implantation in high-risk patients with severe aortic stenosis: the U.K. TAVI (United Kingdom Transcatheter Aortic Valve Implantation) registry. J Am Coll Cardiol. 2011;58:2130-8.

9. Hahn RT, Pibarot P, Stewart WJ, Weissman NJ, Gopalakrishnan D, Keane MG, et al. Comparison of transcatheter and surgical aortic valve replacement in severe aortic stenosis: a longitudinal study of echocardiography parameters in cohort a of the PARTNER trial (placement of aortic transcatheter valves). J Am Coll Cardiol. 2013;61:2514-21.
10. Tamburino C, Capodanno D, Ramondo A, Petronio AS, Ettori F, Santoro G, et al. Incidence and predictors of early and late mortality after transcatheter aortic valve implantation in 663 patients with severe aortic stenosis. Circulation. 2011;123:299-308.

11. Gotzmann M, Pljakic A, Bojara W, Lindstaedt M, Ewers A, Germing A, et al. Transcatheter aortic valve implantation in patients with severe symptomatic aortic valve stenosis-predictors of mortality and poor treatment response. Am Heart J. 2011;162:238-45.e231.

12. Athappan G, Patvardhan E, Tuzcu EM, Svensson LG, Lemos PA, Fraccaro C, et al. Incidence, predictors, and outcomes of aortic regurgitation after transcatheter aortic valve replacement: meta-analysis and systematic review of literature. J Am Coll Cardiol. 2013;61:1585-95.

13. Masri A, Schoenhagen P, Svensson L, Kapadia SR, Griffin BP, Tuzcu EM, et al. Dynamic characterization of aortic annulus geometry and morphology with multimodality imaging: predictive value for aortic regurgitation after transcatheter aortic valve replacement. J Thorac Cardiovasc Surg. 2014; 147:1847-54.

14. Jerez-Valero M, Urena M, Webb JG, Tamburino C, Munoz-Garcia AJ, Cheema A, et al. Clinical impact of aortic regurgitation after transcatheter aortic valve replacement: insights into the degree and acuteness of presentation. JACC Cardiovasc Interv. 2014;7:1022-32.

15. Kodali S, Pibarot P, Douglas PS, Williams M, Xu K, Thourani V, et al. Paravalvular regurgitation after transcatheter aortic valve replacement with the Edwards Sapien valve in the PARTNER trial: characterizing patients and impact on outcomes. Eur Heart J. 2015;36:449-56.

16. Hahn RT, Pibarot P, Weissman NJ, Rodriguez L, Jaber WA. Assessment of paravalvular aortic regurgitation after transcatheter aortic valve replacement: intra-core laboratory variability. J Am Soc Echocardiogr. 2015;28: 415-22.

17. Zoghbi WA, Chambers JB, Dumesnil JG, Foster E, Gottdiener JS, Grayburn PA, et al. Recommendations for evaluation of prosthetic valves with echocardiography and Doppler ultrasound: a report from the American Society of Echocardiography's Guidelines and Standards Committee and the Task Force on Prosthetic Valves, developed in conjunction with the American College of Cardiology Cardiovascular Imaging Committee, Cardiac Imaging Committee of the American Heart Association, the European Association of Echocardiography, a registered branch of the European Society of Cardiology, the Japanese Society of Echocardiography and the Canadian Society of Echocardiography, endorsed by the American College of Cardiology Foundation, American Heart Association, European Association of Echocardiography, a registered branch of the European Society of Cardiology, the Japanese Society of Echocardiography, and Canadian Society of Echocardiography. J Am Soc Echocardiogr. 2009;22:975-1014. quiz 1082-14.

18. Lancellotti P, Tribouilloy C, Hagendorff A, Moura L, Popescu BA, Agricola E, et al. European association of echocardiography recommendations for the assessment of valvular regurgitation. Part 1: Aortic and pulmonary regurgitation (native valve disease). Eur J Echocardiogr. 2010;11:223-44.

19. Kappetein AP, Head SJ, Genereux P, Piazza N, van Mieghem NM, Blackstone EH, et al. Updated standardized endpoint definitions for transcatheter aortic valve implantation: the Valve Academic Research Consortium-2 consensus document. J Am Coll Cardiol. 2012;60:1438-54.

20. Pibarot P, Hahn RT, Weissman NJ, Monaghan MJ. Assessment of paravalvular regurgitation following TAVR: a proposal of unifying grading scheme. JACC Cardiovasc Imaging. 2015;8:340-60.

21. Dahiya A, Bolen M, Grimm RA, Rodriguez LL, Thomas JD, Marwick TH. Development of a consensus document to improve multireader concordance and accuracy of aortic regurgitation severity grading by echocardiography versus cardiac magnetic resonance imaging. Am J Cardiol. 2012;110: 709-14.

22. Hahn RT, Little SH, Monaghan MJ, Kodali SK, Williams M, Leon MB, et al. Recommendations for comprehensive intraprocedural echocardiographic imaging during TAVR. JACC Cardiovasc Imaging. 2015;8:261-87.

Key Words: aortic regurgitation, paravalvular regurgitation, transcatheter aortic valve, transcatheter aortic valve implantation, transcatheter aortic valve replacement 